\title{
Evaluation of hind foot score of Pirani scoring system in treatment of congenital talipes equinovarus by Ponseti method
}

\author{
Menon P.G. ${ }^{1}$, Thokaloath R.S. ${ }^{2}$ \\ ${ }^{1}$ Dr. P. Gopinath Menon, Professor, Department of Orthopaedics, Sri Ramachandra Medical College and Research \\ Institute Porur, Chennai, ${ }^{2}$ Dr. Rahul Sreenivasan Thokaloath, DNB Orthopaedics, Lecturer, Orthopaedics, Government \\ Medical College Kottayam, Kerala, India.
}

Corresponding Author: Dr. Rahul Sreenivasan Thokaloath, D. Orth, DNB (Orth), lecturer in Orthopaedics Kottayam Government Medical College, Kerala, Email: rahul.thokaloath@gmail.com

\begin{abstract}
Background: Congenital Talipes Equino Varus (CTEV) is a congenital complex deformity. Pirani scoring system is most commonly used for classification. In the Ponseti technique of management, those undergoing tenotomy had higher hindfoot score compared to the non-tenotomy group. Hence evaluation of the factor in hindfoot score of the Pirani scoring system, which can predict the need for tenotomy later is important. Materials and Methods: Hind Foot Score of Pirani Scoring System in the treatment of CTEV by Ponseti Method of serial manipulative corrective casting was evaluated on 40 Infants (up to 1 year of age) with 59 idiopathic clubfeet presenting to orthopedic surgery department of Sri Ramachandra medical college and research institute between June 2010 to June 2012. Results: 51 out of the 59 clubfeet (40 patients) underwent tenotomy [86\%]. The mean initial modified Pirani score in the tenotomy group was 4.90 , and in a non-tenotomy group, it was $2.44(\mathrm{p}<0.005)$. The mean hindfoot score in tenotomy and the non-tenotomy group was 2.70 and 1.38 respectively $(\mathrm{p}<0.005)$. All children with the rigidity of equinus less than one were corrected by serial casting alone. $98 \%$ of clubfeet with the rigidity of equinus score 1 underwent tenotomy. The combination of the severity of posterior crease and rigidity of hindfoot showed $100 \%$ with maximum score 2 underwent tenotomy while all below 1.5 scores got corrected by casting alone. Conclusions: Initial rigidity of equinus and severity of posterior crease of hindfoot score of the Pirani scoring system help us in predicting the need for tenotomy later. Combined score of the rigidity of equinus and severity of posterior crease can predict the need for tenotomy better than the emptiness of heel combinations. As the equinus deformity increases to a maximum and posterior crease become severe, then tenotomy is required. Level of evidence: Level 1 High-quality prospective study. The study was started before the first patient enrolled. All patients were enrolled at the same point in their disease with $\geq 80 \%$ follow-up of enrolled patients.
\end{abstract}

Keywords: Congenital Talipes EquinoVarus (CTEV), Clubfoot, Ponseti Method, Pirani Score, Hind Foot Score

\section{Introduction}

Congenital Talipes Equino Varus (CTEV) also known as Congenital Clubfoot is a congenital complex deformity which is typically diagnosed immediately after birth[1, 2]. It has four components- Hindfoot Equinus, Hindfoot Varus, Forefoot Adductus and Midfoot Cavus [1-3]. The initial management should be non-surgical and started as soon as possible after birth $[2,4]$. A variety of manipulations, splinting, strapping, bracing, and casting techniques have been advocated in an attempt to achieve correction of the deformity[3]. But results with non-surgical methods have often been less than optimal, with partial corrections, recurrence

Manuscript Received: $11^{\text {th }}$ September 2018

Reviewed: $20^{\text {th }}$ September 2018

Author Corrected: $26^{\text {th }}$ September 2018

Accepted for Publication: $30^{\text {th }}$ September 2018 and other complications $[3,5]$. In the pre-Ponseti era, management was based on conservative treatment followed by operative treatment if failed. The Ponseti technique is essentially conservative [6]. The Ponseti method comprises a series of manipulations and immobilizations, as well as Achilles tenotomy.

Then an orthosis is used after tenotomy, for sustaining the correction attained and to prevent recurrence. Clinical assessment has been the oldest method of assessing the deformity. Classification systems that are accepted worldwide are the Dimeglioet al [7], Pirani [8, 9] and International Clubfoot Study Group (ICFSG) classification system.Pirani Score assesses the level of severity of each of the components of Clubfoot 


\section{Original Research Article}

effectively, conveniently and easily [8,9]. The family of the children starting with Ponseti treatment usually enquire the surgeon about the need for tenotomy and the number of casts. Pirani scoring system has been considered as an ideal answer for these questions by several authors[10, 11]. Those undergoing tenotomy had higher hindfoot score compared to the nontenotomy group[10, 12]. Hindfoot score consists of the severity of posterior crease, the rigidity of equinus and emptiness of heel. Some patients with medium hindfoot score had undergone tenotomy $[10,12]$. Hence we decided to find out the important component of hindfoot score of the Pirani scoring system $[8,9]$ that can predict the need for tenotomy later.

\section{Materials and Methods}

Place of study: the study was conducted in the department of orthopaedic surgery Sri Ramachandra medical college and research institute.

Type of study: The study was a prospective observational study

Study population: Children with clubfeet brought to the outpatient section of the study setting, who had undergone treatment through the ponseti method of serial manipulative corrective casting were considered as study population.

Sample size \& Sampling method: A total of 40 children with 59 club feet reporting during the study recruitment period who had satisfied the inclusion and exclusion criteria were enrolled by Universal sampling

Study period: The data collection for the study was conducted between June 2010 to June 2012

Inclusion criteria: Our inclusion criteria were newborn and children up to 1 year of age with idiopathic clubfoot.

Exclusion criteria: We excluded children greater than one year of age at the time of the first visit, children previously treated for clubfoot, Postural clubfoot, Clubfoot associated with neuromuscular disease syndromes and chromosomal aberration.

Study procedure: Initial severity of congenital talipes equinovarus was assessed by the modified Pirani scoring system. [8, 9]. The hind foot score of pirani scoring system consists of 3 components severity of posterior crease, the rigidity of equinus and emptiness of heel. The severity of posterior crease was graded as score 0 when multiple fine creases, score 0.5 when 1 or 2 deep creases and score 1 when deep creases change the contour of the arch. The rigidity of equinus was graded as score 0 when normal ankle dorsiflexion, score 0.5 when dorsiflexes but not fully and score 1 when cannot dorsiflex to neutral.

The emptiness of heel was graded as score 0 when calcaneal tuberosity easily palpable, score 0.5 when 1 or 2 deep creases and score 1 when heel not palpable.

Hip and spine were clinically examined for anomalies. Lateral and medial sole striking test was done to detect any neuromuscular imbalance. Deformity correction is started by ponseti technique [6]. The initial cast was applied with the forefoot inverted and the first ray elevated to correct the cavus deformity.

Every week cast was changed, and manipulation was done for half an hour by us before applying next cast. Rest of the casts was applied while gently abducting the forefoot, navicular, and cuboid around the talus, allowing correction of the adducts as well as the heel varus.

The final cast was applied with the foot in $15^{\circ}$ of dorsiflexion. In most cases, tendoachilles was very tight, and stretching was not possible. In these cases, percutaneous tendoachilles tenotomy was performed in operation theatre under local anaesthesia.

After achieving 15 degrees or more dorsiflexion, last plaster is given with foot in $70^{\circ}$ of abduction for three weeks. After removing casting final Pirani score was assessed and foot abduction orthosis with $70^{\circ}$ external rotation of the affected foot and a $15^{\circ}$ bend of the connecting bar is given for constant use (at least 23 hours per day) for the next four months or till the child walks.

Skin abrasions due to rubbing of the edges of the casts in 3 cases were managed by leaving the area hygienically open for a few days and application of antibiotic cream locally. In these cases, the casting resumed once the skin lesions healed.

Statistical methods: Descriptive analysis was done by mean and standard deviation for quantitative variables, frequency and proportion for categorical variables. The quantitative variables were compared between tenotomy and non tenotomy group using independent sample ttest. $\mathrm{P}$ Value $<0.05$ was considered as statistically significant 


\section{Original Research Article}

\section{Results}

Table-1: Comparison of mean values in tenotomy and non-tenotomy group.

\begin{tabular}{|c|c|c|c|}
\hline \multirow{2}{*}{ Parameter } & \multicolumn{2}{|c|}{ Tenotomy (Mean \pm SD) } & \multirow{2}{*}{ P value } \\
\cline { 2 - 3 } & Done $(\mathbf{N}=\mathbf{5 1})$ & Not done (N=8) & \\
\hline Initial Pirani score & $4.9 \pm 1.08$ & $2.44 \pm 0.78$ & $<0.001$ \\
\hline Final Pirani score & $0.12 \pm 0.21$ & $0.06 \pm 0.18$ & 0.493 \\
\hline Mid foot score & $2.20 \pm 0.74$ & $1.06 \pm 0.32$ & $<0.001$ \\
\hline Hind foot score & $2.70 \pm 0.43$ & $1.38 \pm 0.58$ & $<0.001$ \\
\hline
\end{tabular}

The mean initial modified Pirani score in the tenotomy group is 4.90, and in a non-tenotomy group, it is $2.44(\mathrm{p}<0.005)$ [table1]. Thus the need for tenotomy is very high when the Pirani score is above 4.5. Final modified Pirani score mean is 0.12 in the tenotomy group and 0.06 in the non-tenotomy group $(\mathrm{p}<0.493)$ which shows that both values are insignificant. Thus the final outcome is the same whether the child undergoes tenotomy or not.

Similarly, the mean hindfoot score in tenotomy and the non-tenotomy group is 2.70 and 1.38 respectively $(\mathrm{p}<0.005)$ which shows it is a significant value [table1]. Hence need of tenotomy to correct equinus is high when the hindfoot score exceeds $2.5 .93 .55 \%$ of the children with the emptiness of heal 1 required tenotomy and even $50 \%$ with the emptiness of heel of 0 underwent tenotomy [graph1]. Hence emptiness of heel can't predict accurately whether tenotomy needed or not. All children with the rigidity of equinus less than 1 are corrected by serial casting alone but in posterior crease severity score of even $0.5,41.6 \%$ undergone tenotomy [graph1]. So the low value of rigidity of equinus predicts better than that of the severity of posterior crease regarding management. Hence if the rigidity of equinus is low initially, the foot can be corrected by serial casting alone. But the rigidity of equinus score one does not say it needs tenotomy as only $98 \%$ of clubfeet with the rigidity of equinus score one undergone tenotomy.

$98 \%$ of the children with the rigidity of equinus 1 and $100 \%$ of children with the severity of posterior score one required tenotomy to correct hindfoot deformity. None of the feet with 0 scores of severity of posterior crease or rigidity of equinus had undergone tenotomy. When the score of each subgroup of hindfoot combined [graph2] the combination of the severity of posterior crease and rigidity of hindfoot shows 100\% of maximum score 2 undergone tenotomy and all below 1.5 scores got corrected by casting alone. Combined score of the rigidity of equinus and severity of posterior crease can predict the need for tenotomy better than the emptiness of heel combinations. Therefore as the equinus deformity increases to a maximum and posterior crease become severe, then tenotomy is required.

The mean no. of cast increases as the hind foot deformity increases. It is more in the case of the tenotomy group as the deformity is more in the tenotomy group (table 2 ).

The final Pirani score depends on the initial degree of hindfoot deformity. When the degree of initial hindfoot deformity is high, the outcome will be poor [graph3].

Table-2: Mean number of casts in a non-tenotomy group.

\begin{tabular}{|c|c|c|c|c|c|c|c|c|c|}
\hline & \multicolumn{3}{|c|}{ Tenotomy group } & \multicolumn{3}{|c|}{ Non-tenotomy } & \multicolumn{3}{|c|}{ Total group } \\
\hline & 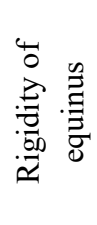 & 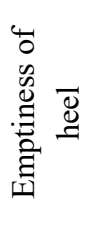 & 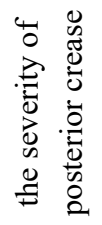 & 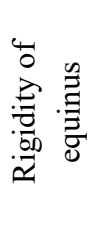 & 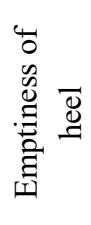 & 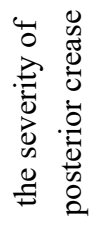 & 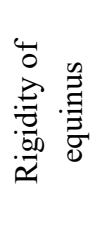 & 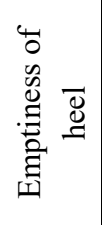 & 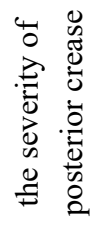 \\
\hline 0 & 0 & 5.75 & 0 & 0 & 4 & 3 & 0 & 4.88 & 3 \\
\hline 0.5 & 0 & 6.5 & 6.6 & 4.71 & 4 & 4.86 & 4.71 & 6.25 & 5.58 \\
\hline 1 & 6.88 & 7.28 & 6.91 & 4 & 6.5 & 0 & 6.83 & 7.23 & 6.91 \\
\hline
\end{tabular}


Original Research Article

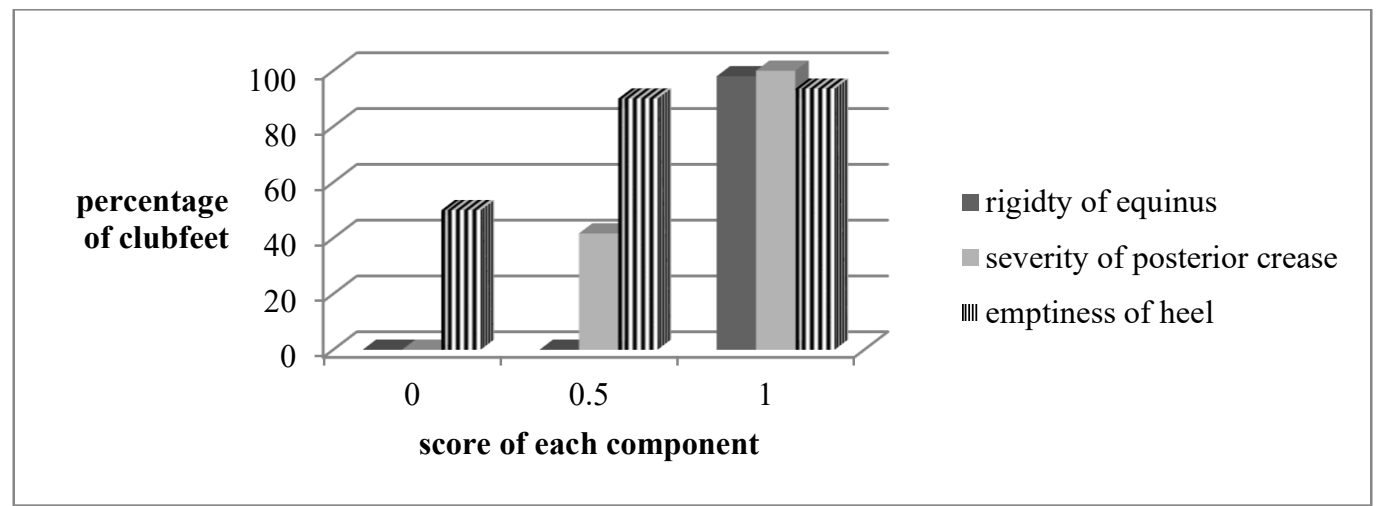

Graph-1: Percentage of club feet undergone tenotomy

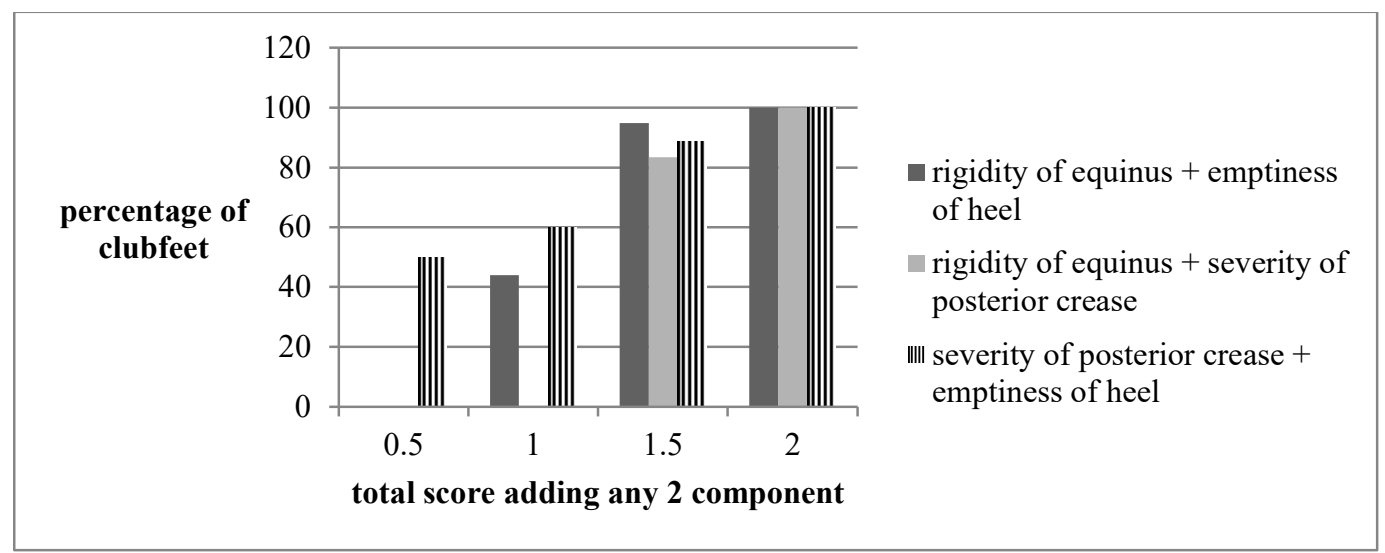

Graph-2: Percentage of club feet undergone tenotomy.

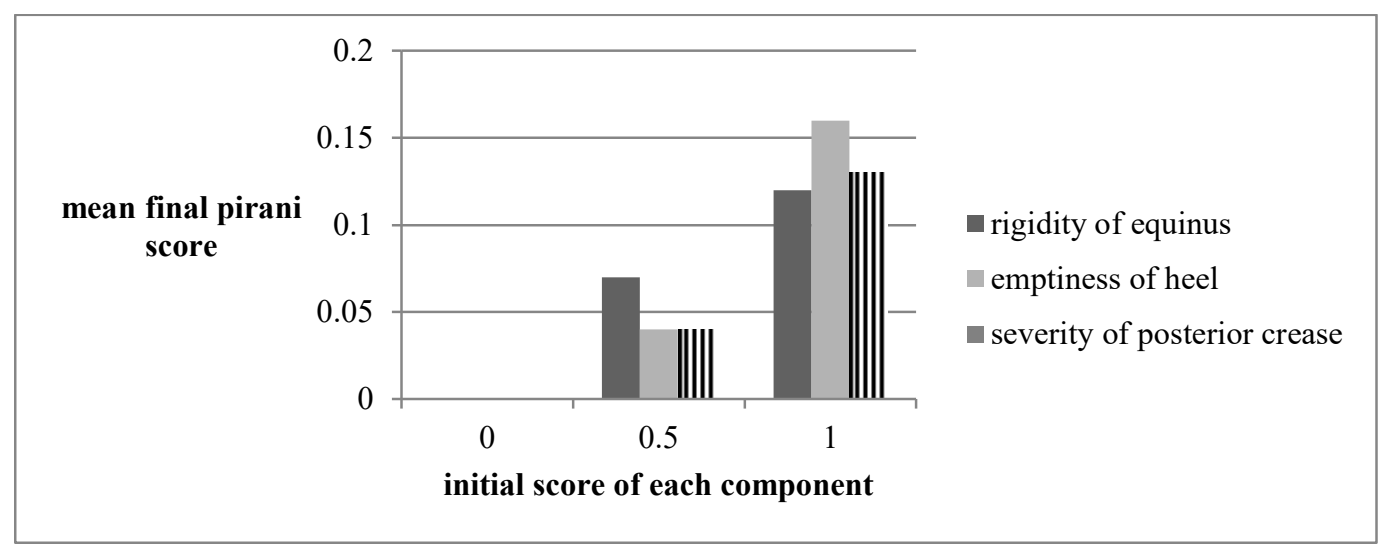

Graph-3: Mean final Pirani score of each foot with initial hindfoot score.

\section{Discussion}

CTEV is a common congenital orthopaedic disorder described by both equinovarus, an excessively turned in foot and CAVUS, a high medial longitudinal arch, which when untreated results in long-term disability, deformity and pain[2]. The primary aim of management is to reduce or eliminate all the CTEV deformity components to obtain cosmetically and functionally acceptable foot with the least possible interruption of the socio-economic conditions of the family in the minimum duration possible. The Ponseti serial corrective cast management $[5,13]$ is an effective, easy and economical method of management where the deformity is corrected by weekly serial corrective cast manipulation. The aim of clubfoot treatment by the Ponseti method is to achieve a corrected foot, with at least $15^{\circ}$ dorsiflexion and $70^{\circ}$ abduction, and fit comfortably into a brace at the recommended setting[14]. 
Original Research Article

Table-3: Comparison of baseline characteristics between authors of various studies.

\begin{tabular}{|c|c|c|c|c|}
\hline & $\begin{array}{c}\text { Dyer PJ and Davis } \\
\mathrm{N}[8]\end{array}$ & $\begin{array}{c}\text { Scher DM et } \\
\mathrm{al}[10]\end{array}$ & $\begin{array}{c}\text { Porecha M and } \\
\text { Parmar D[12] }\end{array}$ & Present study \\
\hline No. of feet & 70 feet(47 cases) & $\begin{array}{c}50 \text { feet }(35 \\
\text { cases })\end{array}$ & 50 feet (30 cases) & 59 feet (40cases) \\
\hline Tenotomy & 42 feet(60\%) & 36 feet (72 \%) & 31 feet $(62 \%)$ & 51 feet $(86 \%)$ \\
\hline Tenotomygroup & 5.31 & 5.7 & $6-9$ & 6.88 \\
\hline $\begin{array}{c}\text { Non- } \\
\text { Tenotomygroup }\end{array}$ & 3.63 & 4.7 & $4-6$ & 4.63 \\
\hline
\end{tabular}

Table-4: Comparison of Pirani Scores.

\begin{tabular}{|c|c|c|}
\hline $\mathbf{2}$ & Dyer PJ and Davis N [8] & Present study \\
\hline \multicolumn{3}{|c|}{ Mean initial total scores } \\
\hline Tenotomy group & 4.96 & 4.90 \\
\hline Non-tenotomy group & 2.16 & 2.44 \\
\hline \multicolumn{2}{|c|}{ Final Pirani score } \\
\hline Tenotomy group & 0.5 & 0.12 \\
\hline Non-tenotomy group & 0.5 & 0.06 \\
\hline
\end{tabular}

Our study results show that the Pirani scoring system can be used to clarify the need for tenotomy and allows an estimate of the number of weekly plaster casts required.Our study objectives and methodology were similar to that of studies done by Dyer PJ and Davis N [8], Scher DM et al[10], Porecha M and Parmar D[12]. Our sample size was similar to that of other previous studies. We did our study on 59CTEV feet while Dyer PJ and Davis N [8] carried out their study on 70 feet. Both Scher DM et al[10], Porecha M and Parmar D [12] carried their study on 50 feet. Similar to our study (82\%), the majority of the study subjects belonged to the tenotomy group in their studies ranging from $60 \%$ to $72 \%$. The mean initial Pirani scores of our study were comparable with that of a study done by Dyer PJ and Davis N[8]. In our study.

We found that the need for tenotomy is very high when the initial Pirani score is above 4.5. There was a significant difference $(\mathrm{p}<0.005)$ in mean initial Pirani score between tenotomy group (4.9) and non-tenotomy group (2.44). Similar to our study, Dyer PJ and Davis N [8] also observed a significant difference $(\mathrm{p}=0.012)$ in mean initial Pirani scores between the tenotomy (4.96) and non-tenotomy (4) groups. They also observed a highly significant difference ( $\mathrm{p}<$ $0.0005)$ in the mean initial hindfoot scores between the two groups (2.81 for the tenotomy versus 2.16 for the nontenotomy group) similar to our study (Hindfoot score in tenotomy and the non-tenotomy group was 2.70 and 1.38 respectively, $\mathrm{p}<0.005$ ). There was no significant difference in final Pirani scores between the groups in our study ( $\mathrm{p}$ $<0.493$ ) indicating that the final outcome is the same whether the child undergoes tenotomy or not.

The Pirani score demonstrates its importance with regards to assessing the severity of clubfoot, mainly at a presentation in an unoperated congenital clubfoot less than two years of age and then at progress. The Pirani scoring system works by assessing six clinical signs of contracture, which may score 0 (no deformity), 0.5 (moderate deformity) or 1(severe deformity) $[8,9]$. The six signs are separated into three related to the hindfoot (severity of the posterior crease, the emptiness of the heel and rigidity of the equinus), and three related to the midfoot. Thus, each foot can receive a hindfoot score between 0 and 3, a midfoot score between 0 and 3 and a total score between 0 and 6 . The total score is recorded after every visit. Pirani scoring is known to be valid and reliable for providing a good forecast about the potential treatment for an individual foot, such that a higher score at presentation may indicate the requirement of a higher number of casts to correct the deformity[8]. A Pirani score of six means the most severe deformity and the Pirani score of zero would be a corrected clubfoot [15]. It allows the treating practitioner to know how the patient is responding to treatment, to know when tenotomy is indicated, and to reassure parents regarding progress.

Dyer PJ and Davis N [8] in their study observed the mean number of casts was 5.31 for the tenotomy group and 3.63 for the non-tenotomy group. The former required significantly more plasters $(\mathrm{p}<0.0005)$. Similarly, in our study, it was 6.88 


\section{Original Research Article}

for the tenotomy group and 4.63 for the non-tenotomy group. Porecha $\mathrm{M}$ and Parmar $\mathrm{D}[12]$ also observed a higher mean number of casts in the tenotomy group.The family of the children starting with Ponseti treatment usually enquire the surgeon about the need for tenotomy and the number of casts. Pirani scoring system has been considered as an ideal answer for these questions by several authors $[10,11]$. When assessed for interobserver reliability the kappa score showed Pirani scoring to be almost perfect and much better than any previous scoring system $[9,16]$. Local factors in an idiopathicclubfoot can be better assessed than that in a patient with specific cause for clubfoot[14, 17]. Scher et al[10] compared Pirani et al[9] and Dimeglio et al[7] scores with the need for a tenotomy. For both systems, they established a link between a high-scoring foot and the need for tenotomy. Parents whose children are starting Ponseti treatment are likely to inquire whether a tenotomy will be required. Scher's study suggests that the Pirani system would answer this question. Pirani score would also allow the surgeon to give more specific advice on a number of casts required while other studies did not individually analyse the predicting factors of Hindfoot score, we did so.

Hindfoot score consists of the severity of posterior crease, the rigidity of equinus and emptiness of heel. Some patients with medium hindfoot score had undergone tenotomy [10,12]. In our study emptiness of heel couldn't predict accurately whether tenotomy was needed or not as seen in graph 1 and also the low value of rigidity of equinus predicts better than that of the severity of posterior crease regarding management. In our study, a combined score of the rigidity of equinus and severity of posterior crease predicted the need for tenotomy better than the emptiness of heel combinations. Therefore as the equinus deformity increases to a maximum and posterior crease become severe, then tenotomy is definitely required. Some patients with medium hindfoot score had undergone tenotomy $[10,12]$ in other studies.

Dyer PJ and Davis N [8] observed a significant positive correlation between the initial Pirani score and number of casts required in their study. A foot with a hindfoot score of 2.5 or 3 has a $72 \%$ chance of requiring a tenotomy. Similarly, in our study, the mean no. of cast increases as the hind foot deformity increases. It is more in the case of the tenotomy group as the deformity is more in the tenotomy group as shown in table2, $3 \& 4$. Other authors reported that those undergoing tenotomy had higher hindfoot score compared to the non-tenotomy group [10, 12].

Aggarwal A et al[11] in their study also observed that the more severe the initial deformity higher Pirani Score, the more casts were required to obtain correction and that age at initial presentation, quality (mobility) of foot and Pirani Score atpresentation, has a direct bearing on final results. Scher DM et al[10]. also observed that rigidity of the foot, and not just the overall severity of the initial equinus, is an important factor in predicting the need for a tenotomy.

Limitations: Our study is limited by the fact that the true functional outcome of these two groups cannot be determined until the child has completed growth. Pirani score also does not reflect the critical transition adequately from the treatment phase of casting into the maintenance phase of bracing in all patients.

\section{Conclusion}

To conclude, as the equinus deformity increases to a maximum and posterior crease become severe then tenotomy is definitely required. Initial hindfoot score influences the final Pirani score. Combined score of the rigidity of equinus and severity of posterior crease can predict the need for tenotomy better than the emptiness of heel combinations.

So it is recommended that as the equinus deformity increases to a maximum and posterior crease become severe, then tenotomy is required. With non surgical procedures giving non satisfactory results, it is recommended that surgical procedures should be advocated early based on the pirani score. Management should focus mainly on eliminating and reducing deformity to obtain a cosmetically and functionally acceptable foot with the least possible interruption.
What this study adds to existing Knowledge? Previous studies have demonstrated that those undergoing tenotomy had higher hindfoot score compared to the non-tenotomy group. Our study adds that initial rigidity of equinus and severity of posterior crease of hindfoot score of the Pirani scoring system help us in predicting the need for tenotomy later. Our study also adds to this knowledge thatcombined score of the rigidity of equinus and severity of posterior crease can predict the need for tenotomy better than the emptiness of heel combinations.

In this study, protocol preparation and getting approval from the ethical committee was done by the first author. The corresponding author did the data collection, analysis and writing of the manuscript. Proof reading and editing was also done by the corresponding author. 


\section{Original Research Article}

There was no external support or sponsors or conflict of interest in this study.

Conflict of interest: None declared.

\section{Funding: Nil, Permission from IRB: Yes}

\section{References}

1. Cummings RJ, Davidson RS, Armstrong PF, Lehman WB. et al. Congenital clubfoot. Instr Course Lect. 2002; 51: 385-400.

2. Gray K, Pacey V, Gibbons P, et al. Interventions for congenital talipes equinovarus (clubfoot). Cochrane Database Syst Rev. 2014 Aug 12;(8):CD008602. doi: 10.1002/14651858.CD008602.pub3.

3. Anand A, Sala DA. Clubfoot: etiology and treatment. Indian J Orthop. 2008 Jan;42(1):22-8. doi: 10. 4103/ 0019-5413.38576.

4. Kite JH. Nonoperative treatment of congenital clubfoot. Clin OrthopRelat Res. 1972 May;84:29-38.

5. Ponseti IV, Smoley EN. The classic: congenital club foot: the results of treatment. 1963. Clin OrthopRelat Res. 2009 May;467(5):1133-45. doi: 10.1007/s11999009-0720-2. Epub 2009 Feb 14.

6. Brand RA. Clubfoot: etiology and treatment Ignacio V. Ponseti, MD, 1914-. Clin OrthopRelat Res. 2009 May; 467(5):1121-3. doi: 10.1007/s11999-009-0719-8. Epub 2009 Jan 30.

7. Diméglio A, Bensahel $\mathrm{H}$, Souchet $\mathrm{P}$, et al. Classification of clubfoot. J PediatrOrthop B. 1995; 4 (2):129-36.

8. Dyer PJ, Davis N. The role of the Pirani scoring system in the management of club foot by the Ponseti method. J Bone Joint Surg Br. 2006 Aug; 88 (8):10824. doi:10.1302/0301-620X.88B8.17482
9. Pirani S, Outerbridge H, Sawatzky B, Stothers K. A reliable method of clinically evaluating a virgin clubfoot evaluation. 21st SICOT congress. 1999;29:2-30

10. Scher DM, Feldman DS, van Bosse HJ, et al. Predicting the need for tenotomy in the Ponseti method for correction of clubfeet. J PediatrOrthop. 2004 JulAug;24(4):349-52

11. Aggarwal A, Gupta N. The Role of Pirani Scoring System in the Management and Outcome of Idiopathic Club Foot by Ponseti Method. IJSR. 2016;5(6):1284-7.

12. David BH, Olayinka O A, Oluwadare E, et al. Predictive value of Pirani scoring system for tenotomy in the management of idiopathic clubfoot. J Orthop Surg (Hong Kong). 2017 May-Aug; 25 (2): 2309499017713896. doi: 10.1177/2309499017713896.

13. Chueire AJ, Carvalho Filho G, Kobayashi OY, Carrenho L. Treatment of congenital clubfoot using Ponseti method. Rev Bras Ortop. 2016 May 4;51 (3): 313-8. doi: 10.1016/j.rboe.2015.06.020. eCollection 2016 May-Jun.

14. Ponseti IV. Treatment of congenital club foot. J Bone Joint Surg Am. 1992 Mar;74(3):448-54.

15. Dobbs MB, Nunley R, Schoenecker PL Long-term follow-up of patients with clubfeet treated with extensive soft-tissue release. J Bone Joint Surg Am. 2006 May; 88(5):986-96. doi:10.2106/JBJS.E.00114.

16. Wainwright AM, Auld T, Benson MK, Theologis TN. The classification of congenital talipes equinovarus. J Bone Joint Surg Br. 2002 Sep;84 (7): 1020-4.

17. Cooper DM, Dietz FR. Treatment of idiopathic clubfoot. A thirty-year follow-up note. J Bone Joint Surg Am. 1995 Oct;77(10):1477-89.

\section{How to cite this article?}

Menon P.G, Thokaloath R.S. Evaluation of hind foot score of Pirani scoring system in treatment of congenital talipes equinovarus by Ponseti method. Surgical Update: Int J surg Orthopedics.2018;4(3):126-132.doi:10.17511/ ijoso.2018.i03.06. 